\title{
ABORDAJE SANITARIO DEL EMBARAZO Y LA MATERNIDAD EN LA ADOLESCENCIA. PERSPECTIVA DE LOS PROFESIONALES DE LA SALUD QUE TRABAJAN EN UN CENTRO DE SALUD DE LA CIUDAD DE MONTEVIDEO*
}

\section{HEALTHCARE ASSISTANCE IN EARLY PREGNANCY AND EARLY MOTHERHOOD. HEALTH PROFESSIONAL'S PERSPECTIVES WHO ARE SERVING AT A HEALTH CENTER IN MONTEVIDEO}

\author{
María Martina Silva Laguardia \\ Universidad Católica del Uruguay \\ Loreto Leiva Bahamondes \\ Universidad de Chile
}

\begin{abstract}
Resumen: El artículo contiene el análisis y la reflexión sobre los discursos de profesionales de la salud que trabajan en la asistencia y acompañamiento de adolescentes embarazadas y madres, usuarias de un centro de salud ubicado en la ciudad de Montevideo. El estudio se enmarcó dentro del enfoque cualitativo y fue de corte transversal. La muestra fue intencionada e incluyó a 10 operadores de la salud. De acuerdo al análisis de contenido de las entrevistas realizadas, se intentó caracterizar y comprender el componente intersubjetivo general conformado a partir del encuentro asistencial entre el profesional de la salud y la adolescente. La información brindada por los participantes permitió, además, elaborar una serie de sugerencias que podrían beneficiar el entramado vincular entre ambos, siendo éste, el escenario donde se juega el logro de los desafíos propuestos por el modelo de atención integral de salud para adolescentes.
\end{abstract}

Palabras Clave: embarazo adolescente, maternidad adolescente, asistencia sanitaria

\begin{abstract}
This paper analyzes the discourse of health professionals who assist and support pregnant adolescents and adolescent mothers at a health care center in Montevideo. Cross-sectional data were gathered and analyzed using a qualitative approach. Ten (10) study participants were recruited among healthcare professionals using intentional sampling techniques. Content analysis of the interviews helped in the characterization and understanding of the general intersubject component built upon the care relationship between the health practitioner and the pregnant adolescents and adolescent mothers. The collected data allowed the elaboration of a set of suggestions which could benefit this relationship, a critical element of the adolescents' comprehensive health care model.
\end{abstract}

Keywords: early pregnancy, early motherhood, healthcare assistance

\begin{abstract}
* El estudio de campo fue realizado por María Martina Silva Laguardia en el marco de la tesis de Maestría en Psicología Comunitaria (Universidad de Chile), la cual fue tutoreada por la Dra. Loreto Leiva Bahamondes. La formación académica recibida fue financiada por el Programa de Apoyo para el Desarrollo de Liderazgos Católicos (Prolic Sur) durante el periodo $2010-2011$.
\end{abstract}

\section{Introducción}

Etimológicamente, adolescente proviene del latín adolescens, participio presente del verbo latino adolescere que significa literalmente: estar creciendo (Amorín, Carril y Varela, 2006). Por su parte, Stassen (2004, p.431) señala que la adolescencia es " $E I$ periodo de transición biológica, cognitiva y psicosocial desde la niñez hasta la adultez, que por lo general dura una década o más". A los efectos del trabajo que se presenta, se establecerá como criterio operacional el parámetro cronológico definido por la Organización Mundial de la Salud (OMS, 1990) en Ministerio de Salud Pública (MSP, 2007), la cual establece que la adolescencia es el periodo comprendido entre los 10 a los 19 años de edad. 
Según constata la Comisión Económica para América Latina (CEPAL, 1995, 2002, 2006, 2008), el embarazo y la maternidad en la adolescencia han sido ampliamente estudiados a nivel regional e internacional. La evidencia empírica acumulada sobre el fenómeno, así como también, el compromiso creciente de los Estados en definir políticas y programas de intervención cuya prioridad fuese evitar el embarazo temprano, determinaron su conceptualización en términos de problema sanitario y social (CEPAL, 2008).

En Uruguay, a partir de la década del noventa, las políticas sanitarias concebidas priorizaron acciones derivadas principalmente de un enfoque preventivo, con la intención de reducir la tasa de fecundidad adolescente registrada (Pereira, 2011a). Los resultados obtenidos comprueban la eficacia de las medidas instrumentadas, ya que según los recientes registros nacionales, la tasa de fecundidad adolescente ha descendido (Gerstenblüth, Ferre, Rossi y Triunfo, 2009). No obstante, Setaro y Koolhaas (2008) explican, que la declinación en la fecundidad adolescente debe ser interpretada dentro de la tendencia demográfica que muestra Uruguay. En tal sentido, se observa un descenso en los valores correspondientes a cada grupo de edad, lo cual incide en la tasa global de fecundidad y en las tasas específicas por edad. Esto significa, que si bien la tasa de fecundidad adolescente ha descendido, la misma no ha sido mayor a la observada en mujeres que se encuentran en la cumbre de la fecundidad, es decir entre 20 y 29 años de edad.

Según las últimas cifras oficiales, correspondientes al año 2010, los nacimientos de madres con edades comprendidas entre los 15 y 19 años en el país fue de 7270, representando el $17,4 \%$ del total de nacimientos a nivel nacional. Si se observa la prevalencia según zona geográfica, se registró mayor cantidad de nacimientos en la ciudad de Montevideo (3687) que en el Interior del país (3583). Con respecto al servicio de salud referido, de acuerdo a los datos registrados en la capital del país, se identificó un mayor porcentaje de nacimientos en los pertenecientes a la órbita pública $(24,2 \%)$ que aquellos sucedidos en los centros privados (8\%) (MSP, 2011).

Si bien el embarazo y la maternidad en la adolescencia, se constituyen como fenómenos sociales complejos, en el cual diversos factores parecen intervenir en su expresión (Amorín et al., 2006; Duncan, 2007; Leiva, 2010; Reis Brandao, 2006), actualmente es desde el área de la salud donde se pueden identificar la mayor cantidad de políticas y programas derivados para atender las necesidades psicosociales de este sector de la población (Peregalli, Ubal, Méndez, 2011; Pérez, Silva y Escobar, 2010). Es por ello, que se decidió continuar profundizando al respecto, ya que se considera que la visión parcializada que esto representa, no solamente compromete socialmente de manera unilateral a un solo sector del Estado, sino que probablemente esto comprometa negativamente en sus acciones de intervención a nivel territorial.

El Sistema Nacional Integrado de Salud, cuenta en su estructura con Instituciones de Salud, las cuales operan a nivel público y a nivel privado. El usuario elige libremente cuál de los prestadores de salud prefiere para recibir su atención, al cual accede de acuerdo a su capacidad contributiva. En tal sentido, el Seguro Social de salud, intenta equilibrar la justicia contributiva, la eficacia sanitaria, y la eficiencia económica. En este marco, se define como política de salud el fortalecimiento de los servicios públicos de salud, reposicionando a la Administración de Servicios de Salud del Estado (ASSE) como su prestador principal en el primer nivel de atención, liderando el cambio de modelo de atención a nivel nacional (Setaro y Koolhaas, 2008). La descentralización de los servicios sanitarios públicos formo parte de los cambios instrumentados para tales fines. En tal sentido, el modelo de atención definido por la Red de Atención Primaria (RAP) divide a la capital montevideana en tres regiones: Oeste, Sur y Este. Cada una se encuentra subdividida en zonas, donde se hallan los centros de salud y las policlínicas barriales dependientes (Ávila y Tancredi, 2006).

El centro de salud elegido para conformar la muestra intencional del estudio que se presenta en el artículo, se ubica en la zona nueve de la Región Este de la ciudad de Montevideo. De este centro dependen doce policlínicas barriales. Su elección fue debido a que atiende a un porcentaje significativo de población adolescente en comparación a otras zonas de Montevideo en la órbita pública, siendo en su mayoría menores de 15 años de edad. Esta condición lo posiciona como uno de los centros asistenciales de la RAP donde se instrumentan gran parte de los programas nacionales destinados a la adolescencia en la capital nacional (MSP, 2007). 
El objetivo general del estudio realizado fue conocer el discurso de los operadores de salud acerca de la atención sanitaria que reciben las adolescentes embarazadas y aquellas que son madres, usuarias de un centro de salud de la ciudad de Montevideo. Los resultados obtenidos, fueron examinados de acuerdo a los parámetros establecidos en el modelo de análisis del Sistema Total de Salud (STS) definido por Saforcada (1999, 2008), el cual es descripto mediante los principios del enfoque ecológico sistémico. La información contenida en el presente artículo, describe los aportes proporcionados por los profesionales de la salud consultados respecto a uno de los componentes implicados en el modelo, el componente intersubjetivo general.

\section{A. Abordaje sanitario del embarazo y la maternidad en la adolescencia}

En el marco de la reforma de salud, impulsada por el gobierno a partir del año 2005, se han desarrollado una serie de programas dirigidos a mejorar la protección social para niños y adolescentes del país. En tal sentido, el $\operatorname{MSP}(2007,2009,2010)$ ha generado diversos programas sanitarios que tienen como eje la atención integral del adolescente, ocupando un lugar central la mejora en la calidad de las estrategias para el abordaje de la salud sexual y reproductiva de los mismos. Parte de la estrategia empleada para tales fines, ha sido a través del fortalecimiento del primer nivel de atención y la complementación entre los prestadores de servicios de salud públicos y privados.

El Programa Nacional de Salud Adolescente (MSP, 2007), integra la nueva propuesta sanitaria, desde un enfoque de derecho y género. Sus directrices se integran y ejecutan desde la dinámica asistencial del Espacio Adolescente en los centros de salud. Es desde éste encuadre, donde se instrumentan las acciones de prevención y acompañamiento al embarazo y la maternidad en la adolescencia.

Según se señala en el documento el embarazo en la adolescencia continúa siendo visualizado como un problema, que al igual que los otros mencionados, limitan las oportunidades de vivir plenamente dicha etapa y compromete su desarrollo futuro como individuo biopsicosocial. La novedad planteada, parece ser la intención de modificar la estrategia de abordaje empleada hasta el momento. Si bien la atención preven- tiva continua siendo la prioridad en el abordaje sanitario, se señala como nuevos lineamientos: la adopción de un enfoque de género, el progresivo involucramiento y participación de los adolescentes, el fortalecimiento de los vínculos y redes sociales, el mejorar el trabajo interinstitucional, así como también, la necesidad de generar soportes que acompañen la transición del adolescente hacía la adultez. Además, se reconoce la adopción de una orientación biopsicosocial, el cual se gesta a partir de un diagnóstico situacional de las necesidades y problemas que presenta el adolescente en la consulta. Se agrega, que las condiciones de la misma deberían realizarse a través de servicios privados, confiables y asequibles, asegurando así, una atención de calidad y amigable (MSP, 2007).

Se piensa que la conjunción de los nuevos lineamientos descriptos, podrían estar definiendo el actual abordaje y acompañamiento de las adolescentes en estado de gravidez o madres, lo cual resulta interesante conocer más allá de lo establecido a nivel programático, ya que parecería trascender la categoría atribuida como problema.

\section{B. Modelo Ecológico Sistémico del Sistema Total de Salud}

Saforcada $(1999,2008)$ propone un modelo de análisis sobre el STS, el cual proporciona una mirada esclarecedora y operativa del conjunto de elementos implicados en el servicio asistencial de una sociedad. Desde su perspectiva, éste "involucra todo lo que una sociedad tiene y hace en relación con este proceso y sus manifestaciones" (Saforcada, 2008, p. 49). En tal sentido, se incluyen en sus componentes los

“...fenómenos, acciones, conocimientos y prácticas que caen dentro e la responsabilidad y el área de incumbencia de las instituciones, los profesionales y los técnicos de la salud avalados por las leyes pertinentes ... de instancias de gobierno ... de instancias legislativas y/o de instancias judiciales" (Saforcada, 1999, p. 43).

De acuerdo a los objetivos perseguidos en el presente trabajo, se analizará con mayor detenimiento el componente intersubjetivo general, el cual es considerado por el autor, uno de los elementos que producen mayor ineficacia e 
ineficiencia en el actual sistema de salud de la mayor parte de los países latinoamericanos. Asimismo, la implicancia de este componente en el contexto definido por la situación que establece el embarazo y la maternidad en la adolescencia respectivamente, resulta clave para identificar las oportunidades de cambio dentro del abordaje sanitario hasta ahora instrumentado.

El componente intersubjetivo general, lo define como el "... espacio virtual que se genera a partir de la interacción cotidiana entre el componente formal (profesionales y técnicos) y el componente informal (usuarios del sistema)". Asimismo, se destaca que es el área donde se da el "... intercambio entre las subjetividades de los integrantes de cada uno de estos dos componentes" (Saforcada, 2008, p. 53-54).

Según Saforcada (2008), en este espacio de intercambio se generan las reacciones afectivas, cognitivas, conductuales, constitutivas del componente intersubjetivo general, las cuales retroalimentan (positiva o negativamente) las estructuras y procesos psicosociales que sostienen a las subjetividades de cada componente (formal e informal). Se considera relevante este último aspecto, porque se coincide con el autor cuando sostiene que la retroalimentación negativa de las cosmovisiones, por ejemplo, sobre embarazo y/o maternidad en la adolescencia, construyen una barrera psicosociocultural que opera de manera negativa en el encuentro con el otro. Sus consecuencias inmediatas, de acuerdo a su perspectiva, son la obstrucción del funcionamiento del subsistema de atención sanitaria, y la consolidación de los aspectos negativos de cada componente. Los efectos perjudiciales que esto conlleva, pueden revertirse si se logra caracterizar las mismas con la finalidad de corregir y superar las trabas que complejizan el intercambio o acuerdos satisfactorios entre ambas partes.

Si bien se ha encontrado una serie de documentos oficiales (MSP, 2007, 2009, 2010) que describen la intencionalidad política de un cambio en la modalidad de atención sanitaria, el éxito de cualquier programa de intervención se juega en el campo concreto de aplicación, donde el establecimiento de un vínculo caracterizado por el respeto y la confianza, es fundamental si se pretende emprender el camino hacía el cambio y la transformación social. Esto podría estar indicando que, más allá de los parámetros sanitarios vigentes o los lineamientos de una política sanitaria determinada, es necesario conocer las opiniones que los actores involucrados presentan.

\section{Método}

\subsection{Instrumentos}

Se utilizó la entrevista semiestructurada como técnica cualitativa de recolección de datos, por ser considerada una herramienta facilitadora en situaciones donde se pretende que los sujetos entrevistados expresen sus puntos de vista sobre determinado problema o experiencia (Hernández, Fernández y Baptista, 2006). Se diseñaron dos guías de entrevistas, las cuales, estaban pautadas de acuerdo al objetivo del estudio. Una de ellas se empleó para las entrevistas con los informantes claves, y la otra, se aplicó en los encuentros con los distintos miembros del equipo de salud del Espacio Adolescente del centro participante.

\subsection{Participantes}

Con la finalidad de asegurar que el objeto de estudio fuese abordado desde diferentes ángulos, se decidió involucrar en la investigación a diversos actores sociales implicados en el fenómeno de estudio. Para ello, se realizaron diez entrevistas en total. Se efectuaron tres entrevistas a informantes claves, quienes fueron elegidos de acuerdo a su rol y vinculación con instituciones estatales relacionadas al sistema de salud uruguayo, y por su experiencia en la dirección o coordinación de programas nacionales destinados a la parentalidad en la adolescencia. En los tres casos, su participación permitió conocer los lineamientos generales presentes en la atención sanitaria dirigida a adolescentes embarazadas o madres, así como también, colaboró con la construcción de un marco de referencia adecuado para la comprensión del quehacer profesional en relación al fenómeno de estudio desde el área de la salud.

Asimismo, participaron del estudio siete profesionales o técnicos que integran el Espacio Adolescente que funciona en el centro de salud convocado, y atienden a adolescentes en estado de gravidez o madres. La muestra fue intencionada debido a que la elección de los participantes se realizó de acuerdo a ciertos criterios que se valoraron como necesarios o convenientes para poder tener una unidad de análisis que reuniera las mayores ventajas posibles para los fines que persigue la inves- 
tigación (Serbia, 2007). Del equipo asistencial participaron la ginecóloga, el pediatra, la partera, la agente socioeducativa, el odontólogo, la nutricionista, y la enfermera. De esta manera se logró cubrir el $100 \%$ de las disciplinas que integran el espacio asistencial dirigido a las adolescentes.

\subsection{Procedimiento}

Considerando las características del estudio, el mismo se enmarcó dentro del enfoque cualitativo y fue de corte transversal. Se empleó un diseño abierto y emergente, lo cual permitió que a medida que avanzaba la investigación se pudiesen incluir nuevas entrevistas a las previamente programadas.

El trabajo de campo se inició con la autorización de la RAP de ASSE. Posteriormente se presentó el proyecto a la Dirección del centro de salud, con la finalidad de obtener su aprobación y así lograr su involucramiento en el mismo. Una vez obtenido su autorización, se acordó el procedimiento de implementación del cronograma de entrevistas, así como también, se definió una lista con los profesionales que integraban el Espacio Adolescente.

La mayoría de los profesionales accedieron a participar, brindando su consentimiento para ello.

Una vez finalizada la investigación y elaborado el informe final, se contactó nuevamente a los participantes del estudio, y se concretaron con ellos las entrevistas de devolución sobre los resultados obtenidos.

\section{Análisis de resultados}

La información recogida, se estructuró de acuerdo al objetivo del estudio respetando las narraciones de los participantes, logrando así, describir sus experiencias de acuerdo a su ópti$\mathrm{ca}$, en su lenguaje y con sus expresiones. Para el análisis de la información, se empleó los criterios definidos por el Análisis de Contenido (Hernández et al., 2006; Miles \& Huberman, 1994). Mediante la aplicación de este procedimiento de análisis, se pudo lograr una descripción sistemática del contenido manifiesto de las entrevistas realizadas. Asimismo, se considera que la triangulación que se logró con los datos que se obtuvieron, a través de las diversas fuentes de información y la participación de diferentes agentes de salud dentro del proceso, permitió alcanzar en la indagación cualitativa una mayor riqueza y profundidad (Hernández et al., 2006). Estos aspectos fueron necesarios para que el estudio adquiriera calidad y rigor.

\section{Resultados}

En el siguiente apartado, se describe la información proporcionada por los profesionales de la salud ${ }^{1}$, a partir de la cual se puede inferir las características del componente intersubjetivo, dinamizador del encuentro entre el componente formal e informal (Saforcada, 1999, 2008). Se considera pertinente resaltar que los datos que se presentan en el artículo corresponden a la perspectiva del componente formal, obteniendo de esta manera, una visión parcial del objetivo de estudio abordado.

El primer subcomponente refiere a los saberes y sus repercusiones en las prácticas asistenciales, las cuales se encuentran afectadas por las directrices definidas por el subcomponente de los referentes válidos en salud.

Al respecto, los operadores de salud identificaron el Espacio Adolescente, como la plataforma donde se ejecutan los programas de salud destinados a la población adolescente en general. En este sector asistencial, se atiende también a las adolescentes embarazadas y quienes son madres. A partir de la instrumentación del mismo, se pudo concretizar el abordaje sanitario de la parentalidad temprana desde una óptica integral.

“... los Espacios Adolescentes... se conformaron en los centros de salud, con más o menos integrantes de distintas disciplinas. Justamente se trata de que el adolescente, varones y mujeres ... que en el caso mío que soy ginecóloga veo básicamente mamás adolescentes y adolescentes en general ... para que puedan tener todo lo que es la integralidad de lo que es el servicio de salud. Tiene odontología, nutricionistas, ginecología, la parte de medicina general... hay una pediatra que ve a los adolescentes que son un poco más chicos ... Y después una figura básica y fundamental que ha sido el aporte del MIDES [Ministerio de

1. En letra cursiva aparecen, textualmente, verbalizaciones de los participantes del estudio, que se consideran ilustrativas de las descripciones que se realizaron. Asimismo, la referencia de la fuente será identificada por el número de entrevista [EN] a los efectos de resguardar la identidad de los informantes. 
Desarrollo Social, N. de A.] a la RAP que son el agente de seguimiento socioeducativo ..." (E10).

“...en recepción cuando hay un chico de 17 años o 18 años para bajo y entre 10 años y medio a 11 años para arriba va directamente a este Espacio Adolescente. Por lo tanto, ese chico va a ser visto por una nutricionista, un médico pediatra, un asistente social, una psicóloga, y un odontólogo... esa es la rutina. ..." (E1).

El enfoque holístico señalado, involucra la tarea coordinada de un equipo multidisciplinario de profesionales pertenecientes a distintas áreas de la salud, así como también, una dinámica funcional diferente a los restantes sectores asistenciales que pretende, entre otros, brindar una respuesta inmediata a la demanda del usuario.

“... Espacio Adolescente es un lugar abierto dos veces por semana ... se atienen sin número ... es decir, que si él o ella tiene una consulta o duda y la resuelve en el momento, y no tiene que esperar ni una semana ni un mes ... y en realidad habla con una persona preparada para eso" (E5).

"Si yo comparo con otros servicios, el Espacio Adolescente tiene 'más aceitado' [más fluido, $N$. de A.] los procesos y es todo más rápido ... y tenés mayor respaldo ..." (E2).

Se mencionó, también, sus tareas asociadas a la realización de talleres informativos en los centros educativos u otras instituciones sociales ubicados en la zona de influencia del centro de salud. No obstante, debido a los escasos recursos humanos que cuentan para cubrir la demanda dentro del centro de salud, aludieron a su baja participación comunitaria en comparación a otros períodos de gestión.

“... El Espacio Adolescente no sólo tiene una tarea asistencial, sino que también, tiene una tarea de salir a la comunidad... estamos yendo a escuelas o a comedores... a las ONG... y eso tal vez lo descuidamos un poco ... tenemos muy poco tiempo y mucha demanda" (E3).

"Y hay mucho menos actividad fuera del centro de salud ... Antes se hacía más cosas de lo que ahora se hace. Un poco, como que se desdibujo, pero seguimos funcionando" (E10).
Respecto a las acciones emprendidas por el sistema público de salud en relación al embarazo y la maternidad en la adolescencia, algunos consultados comentaron que perciben mayores propuestas asistenciales destinadas a este sector de la población en comparación a la cantidad de campañas preventivas asociadas al fenómeno, lo cual valoraron positivamente.

“... se están haciendo más cosas como el Servicio de Atención al Embarazo, Maternidad, Paternidad Adolescente [SAEMPA] o trabajar las agentes socioeducativas desde el comienzo del embarazo ... Creo que se están instrumentando más programas y espacios ..." (E4).

“... por ser adolescente, lo que yo veo es que hay más programas y contención... la embarazada adolescente es bastante contenida en la parte psicológica, beneficiada con ese servicio ... como que se la apoya bastante ..." (E5).

Relacionado con este último aspecto, algunos operadores de salud, hicieron alusión a la imposibilidad que perciben en las adolescentes madres de elaborar proyectos complementarios a su maternidad hasta el primer año de su hijo. No obstante, explicaron que en la consulta abordan estos aspectos de la vida, y en el caso que la usuaria lo desee, se realizan los intentos desde el centro de salud para conectarla a diferentes instituciones u organizaciones de la zona.

"Y como mucho en el foco puesto en su hija o hijo ... Especialmente cuando el bebé nació hace poquito ... ellas proyectarse cero ... eso de volver a estudiar o trabajar no, no, cuando sea más grande ... cuando cumpla el año" (E4).

“... y por suerte con lo que tiene que ver con el Espacio Adolescente, y este acompañamiento de las agentes socioeducativas, son las que la conectan con cursos de computación... hay una casa que van madres adolescentes que se llama Casa Lunas... y en realidad se trata de conectar para que tenga un sostén y para que no sea la única meta de su vida ser mamá..." (E5).

No obstante, discrepan con la persistencia de mensajes negativos y culpabilizadores al momento de abordar las campañas de prevención del embarazo temprano por parte del MSP. 
"Hay que empezar a darle otra mirada. Creo que prevención del embarazo adolescente es como ... culpable, te embarazaste sos culpable ... yo no sé si en realidad hay que prevenir el embarazo adolescente ... creo que no hay que educar en la negativa. Tal vez esté bueno ... hacer educación en proyectos de vida" (E5).

En estos casos, propusieron que el abordaje del embarazo en la adolescencia debería estar orientado con otra perspectiva, priorizando la elaboración de proyectos complementarios a la maternidad, donde la construcción de una familia no sea un obstáculo para la concreción de otros proyectos, como estudiar o trabajar. O también, focalizando las estrategias hacía la población adolescente, que por su edad, al transitar por un embarazo posee mayor riesgo médico.

"No sé si en la prevención, pero si en el día de mañana capaz quiere volver a quedar embarazada, pero tal vez quiera terminar el liceo o hacer un curso o capaz ... tienen otras prioridades y no solo la maternidad. Es decir, evalúa un poco más su abanico de posibilidades ... Que no las corren ..." (E5).

“... en realidad esto de la prevención yo lo pondría más en menores de catorce años, de repente ... tal vez ahí haya más riesgo de prematurez ... en estos bebés" (E4).

Asimismo, se propuso que se debería informar a la población de los servicios que se pueden encontrar en los centros de salud para poder evitar un embarazo no deseado, entre ellos, la accesibilidad que hoy se tiene a diversos métodos anticonceptivos. Se señaló que sí las campañas de prevención fuesen más explícitas, no deberían de existir los espacios de consultoría por casos de interrupción de embarazo.

"Pienso que si se hicieran campañas de prevención del embarazo, un poco más abiertas e insistentes ... darían mejores resultados ... y hablar de la importancia del cuidado y del uso del preservativo ... y que estén en conocimiento de todos los servicios que hay en los centros de salud, porque acá tienen todo gratis y al alcance ... el aborto no es la solución ..." (E10).

"Cuando vienen acá les digo que tienen que saber que existen esas medidas ... aunque to- davía no vayan a tener relaciones ... que sepan qué es un anticonceptivo, qué es un preservativo, y que sepan que en el centro de salud nosotros les vamos a brindar la información y darles las medidas ... uno le trata de dar las herramientas, pero ... me parece que es tarea de todos quienes están en los lugares en donde pueda haber jóvenes de reforzar eso ... más allá del papel que pueda llevar la familia ..." (E3).

Más allá de los señalamientos efectuados por los consultados respecto a las prácticas en salud o acciones efectoras, en general se evaluó que el servicio ofrecido a las usuarias adolescentes ha mejorado. No obstante, se visualizaron carencias a nivel intersectorial, específicamente en relación a recursos y respaldos institucionales. Los participantes hicieron referencia a las experiencias de vida de las adolescentes, donde la pobreza, la violencia doméstica, las privaciones emocionales y materiales, definen un marco asistencial que exige un trabajo intersectorial afianzado y eficaz, el cual no se está dando actualmente.

"Yo creo que muchos desafíos pasan por lo intersectorial, porque a veces es ahí que encontramos un poco de agujeros en lo que tiene que ver los recursos, y muchas veces te sentís trabajando un poco solo ..." (E4).

Con respecto a la percepción que tienen los consultados sobre su trabajo como equipo de salud, señalaron que a pesar de los escasos espacios de reflexión y de instancias de reunión multidisciplinarias, continúan funcionando y apoyándose recíprocamente. En tal sentido, se comentó que cada uno está focalizado en su tarea como especialista, sin que esto afecte la dinámica asistencial del servicio, ya que se conocen y saben que cuentan con el apoyo del otro en caso que sea necesario.

“... hoy está siendo muy difícil poder encontrarnos como equipo y poder tener instancias de reunión ... a veces estás un poco tironeado entre la productividad y la calidad" (E4).

"Seguimos trabajando como individualidades ... pero al mismo tiempo sabemos que allá está fulano que me puede apoyar en determinada circunstancias ... o sea, seguimos tratando de armar los eslabones de lo que es el Espacio Adolescente. Pero no hay propuesta de equipo ..." (E10). 
Por otra parte, plantearon que los cambios de gobierno traen consigo cambios de prioridades, afirmando desconocer cuál es el rumbo que el sistema de salud nacional quiere emprender actualmente. Explicaron que el auge que tuvo, en el periodo de gobierno anterior, el Programa Nacional de Salud Adolescente como otras propuestas, se perdió. Esto se ejemplifico aludiendo a la falta de referentes claros o directrices desde el ministerio.

"Este año ha sido un año muy complicado ... la falta de coordinación a nivel central fue muchísimo a nivel de la RAP. Sí, porque de ella depende coordinar capacitaciones, evaluar, el tener jornadas de intercambio ... de cómo están los otros centros trabajando ... eso es muy importante, y se perdió" (E3).

“... por parte del Ministerio tampoco tenés una buena respuesta o demasiado apoyo, porque a su vez, cambian las referentes de las políticas permanentemente ... entonces uno no tiene de dónde apoyarse .... a veces uno solo o un equipo solo no puede con tanta cuestión ... y más que nada cuando las directivas no son claras ... Son confusas" (E1).

En relación al subcomponente de las estructuras psicosocioculturales, reconocieron que sus aproximaciones a la temática se han matizado a partir del trabajo diario con las adolescentes. La experiencia adquirida les ha permitido percibir como natural un fenómeno que, en sus propios contextos socioculturales, hubiese sido calificado en términos de catastrófico o problema indeseable.

"Acá es una zona que la maternidad tiene un poder muy grande, tanto a nivel de mujeres como de hombres ... todo lo que tiene que ver con el hombre y su masculinidad ... Y bueno en las mujeres también ... el poder del embarazo ..." (E4).

“... lo que veo en este grupo de población, que ese embarazo es su proyecto de vida ... y si uno habla de estudiar ... no estamos hablando de una carrera sino terminar secundaria ... hablas de un proyecto de vida más allá de su maternidad y a veces no existe y es lo que más me inquieta o más me preocupa ... ese otro valor, que para uno es importante ... a veces para ellas no" (E8).
Si bien los consultados expresaron su respeto hacía las decisiones que las adolescentes han tomado en relación a estos aspectos vitales, de acuerdo a sus declaraciones, la mayoría de los participantes entendía que el embarazo y por ende la maternidad, integraban la constelación de comportamientos no esperables para este ciclo vital.

"Siento que .... que es una lástima ... el adolescente tiene que estudiar, este disfrutar la vida ... ir a la playa, ir a bailar ... vivir la juventud ...me queda una lástima bárbara [suspira] ... Para mi hijo no lo querría ..." (E2).

"Siento que ... forma parte de una realización personal equivocada, porque en general uno sabe que en general buscan en un embarazo satisfacer una necesidad de ser ... de valorarse ... es una carga pesada de llevar para ellas, porque siento que no están capacitadas ... porque complica un embarazo hacía esa edad ... desde lo biológico hasta la realización personal, hasta la familia ..." (E3).

La mayoría coincidió que la valoración y significación positiva atribuida por las usuarias al estado de gravidez y la maternidad podría deberse también a la ausencia de oportunidades que tienen los adolescentes que viven en esta zona de la capital montevideana. En tal sentido, se destacó que las limitaciones territoriales, en cuanto a recursos e infraestructura, para poder estudiar o permanecer en un centro educativo, condiciona también la creación de proyectos alternativos a la parentalidad en la población adolescente. Si bien reconocieron el poco apoyo que suelen recibir por parte de las familias, así como la pobre valoración que éstas realizan de la educación, las escasas oportunidades sumadas a la precariedad de las mismas, estarían restringiendo los posibles caminos que estos adolescentes pudieran emprender.

"Siento que muchas veces no hay muchos más proyectos de vida que la maternidad en estos lugares ... si no se pueden generar proyectos palpables de vida, como que parece ser la maternidad el más viable y seguro" (E4).

"Siento que en sí el apoyo familiar que ellas tienen es bastante escaso, entonces en realidad ellas están muy solas. Entonces hasta ellas vienen como muy mal en sí, porque tal vez haya 
sido deseado el embarazo, pero a veces cuesta llegar al adolescente... cuesta que se levanten temprano y vengan a la consulta ... como no tiene apoyo familiar y no tienen a alguien... se olvidan, no lo hacen ..." (E5)

"Creo que lo que falta son proyectos de vida que vayan más allá de la maternidad... Hay que ver que más allá de la maternidad existen cosas, existe mundo..." (E5).

Por otra parte, los consultados sostuvieron por unanimidad, que la usuaria del servicio asistencial vive situaciones de significativa vulnerabilidad. A pesar de su comportamiento adulto, perciben su fragilidad potenciada por la situación en la cual están inmersas. En este contexto de vida, la llegada de un hijo también puede ser significado como un nuevo comienzo o una situación que complejiza aún más sus vidas.

" GGurisas que vamos a su casa y vemos donde vive, y no lo podemos creer! Esas cosas que uno se pregunta cómo hacen para poder sobrevivir y vivir en esas condiciones... chiquilinas con piso de barro, sin aberturas en las casas, se les inundan, una humedad terrible y sin luz. $Y$ bueno es eso lo que te supera ..." (E4).

“... en algunos casos me doy cuenta de la vulnerabilidad que tienen, pero hay muchísimas adolescentes que ya se comportan como adultas... Hay algunas que uno ve la fragilidad y la problemática que están viviendo... bueno uno también tiene cortes y sesgos y te pones en madre también..." (E10).

“... es como que están en esa etapa de niñas pero grandes ... yo tengo madres adolescentes de quince años y cuando quedaron embarazadas tenían catorce ... entonces es difícil. Muchas se fueron de su casa, viven con su pareja ..." (E5).

\section{Discusión}

La información presentada en el apartado anterior, refleja la interdependencia de los subcomponentes descriptos por Saforcada (1999, 2008), siendo necesario su análisis considerando su red de conexiones. En tal sentido, no es posible aislar los contenidos vinculados a los saberes en salud y sus repercusiones en la práctica asistencial, de los lineamientos establecidos por los referentes válidos en salud y sus propuestas de formación y capacitación, ya que estos últimos son generadores de opiniones frente a diferentes temas que ocupan y preocupan a los profesionales de la salud consultados. La dinámica que se genera como consecuencia de las interconexiones que se producen entre los subcomponentes delimitan, por lo tanto, sus actitudes hacía esta población, incidiendo en sus prácticas asistenciales cotidianas.

El embarazo y la maternidad en la adolescencia lo entienden como resultado de decisiones deliberadas, y no únicamente en términos de situación no deseada. A pesar de ello, algunos explicitaron que más allá de las condicionantes culturales implicadas, las cuales debían respetarse y considerarse al momento de trabajar con esta población, visualizaban el embarazo en esa etapa de la vida como una situación de riesgo e inconveniente. En tal sentido, se reconoció las limitaciones encontradas en un ser en desarrollo, como es el adolescente, para hacerse cargo de manera óptima de la crianza de un niño, así como también, se señaló la desventaja adicional que agrega un embarazo a una situación social que, previamente, es difícil y reducida en oportunidades.

No obstante, independientemente de los matices encontrados en las posiciones de los consultados sobre la temática, coincidieron en la importancia de interiorizarse por la historia de vida de la adolescente, por su familia y pareja, por sus sueños y proyectos, para intentar acompañar y alentar aquellos proyectos que fuesen complementarios a su maternidad.

Siguiendo con esta línea de análisis, se aludió a la relevancia de adoptar un enfoque basado en las fortalezas del sujeto, independiente de la edad del usuario, al momento de entablar el vínculo asistencial. Desde esta mirada, surgió la importancia de empoderar a las adolescentes, para que sean conocedoras de sus derechos y pudieran ampliar sus posibilidades para controlar sus vidas. Por ello, motivar e incentivar proyectos complementarios a la maternidad, como estudiar o trabajar, parece ocupar una proporción importante del tiempo destinado a la consulta. Esta intencionalidad, adquiere un particular significado cuando se trata adolescentes que viven en condiciones de alta vulnerabilidad, siendo la maternidad un factor que suele sumar una desventaja adicional a una situación que, previamente, era difícil y reducida en oportunidades (Duncan 2007; Pereira, 2011b). 
Según lo señalaron, sus roles implicaban ser agentes facilitadores de un proceso de acompañamiento lo más holístico posible. Es decir, por un lado, tender redes a la interna del centro de salud intentando garantizar un servicio integral, apoyándose en los recursos que proporcione el Espacio Adolescente y demás áreas asistenciales, y por el otro lado, generar nexos inter-institucionales que permitiesen facilitar la inclusión social de las adolescentes una vez que tuviesen a sus hijos.

Sin embrago, la ejecución de las acciones mencionadas, con frecuencia encontraban sus obstáculos para su concreción. Por un lado, por las progresivas restricciones de los espacios de intercambio multidisciplinarios durante la jornada laboral, y por el otro lado, por los limitados soportes sociales existentes fuera de la órbita de la salud, lo cual limita significativamente las posibilidades de garantizar dicho abordaje integral.

En el marco de este contexto, caracterizado por los recursos limitados y las escasas posibilidades de trabajo en red social, el afianzamiento de las instancias multidisciplinarias parece ser una necesidad imprescindible para superar las barreras que suelen existir en el quehacer asistencial. Asimismo, los encuentros entre colegas, especialmente entre aquellos que trabajan con personas que viven en condiciones tan complejas como las presentadas en este trabajo, podrían auspiciar de protectores saludables de los profesionales de la salud. La contención y apoyo entre pares, en este caso, fortalecería las destrezas intra-grupales y con ello, minimizaría el efecto negativo generado por las carencias y debilidades existentes en el STS.

Por otra parte, los entrevistados aludieron a sus discrepancias y coincidencias respecto a las directrices gubernamentales definidas sobre este fenómeno.

En relación a las primeras, se identificaron discrepancias en relación al enfoque y el contenido de la información comunicada por el MSP al momento de abordar las estrategias de promoción de salud sexual y reproductiva. Por un lado, algunos operadores de salud denunciaron la visión reduccionista sobre la parentalidad temprana que rige a nivel ministerial, calificando las estrategias instrumentadas como inadecuadas a la realidad para la cual fueron creadas. Sostuvieron que la perspectiva de riesgo y sus acciones preventivas derivadas, adoptaban un enfoque negativo y culpabilizador a través de un consistente mensaje respecto a los inconvenientes y la no deseabilidad social atribuida a la misma. Y por el otro lado, los cuestionamientos se encauzaron hacía considerar la información proporcionada a la población en términos de escasa y no explícita. En tal sentido, se sostuvo que las campañas oficiales de promoción de salud sexual reproductiva no comunicaban acerca de los recursos que los usuarios en general podrían encontrar en los centros de salud para evitar un embarazo no deseado.

En cuanto a las coincidencias, los operadores de salud realizaron una valoración positiva de los espacios que desde la salud se han habilitado a favor de la atención integral de la adolescente en estado de gravidez. En esta línea de acción, se reconoció unánimemente el Programa de Agentes Socioeducativos ${ }^{2}$.

No obstante, calificaron de preocupante las escasas propuestas que facilitan la inclusión social de la madre adolescente, más allá de las iniciativas del área de la salud. Desde la perspectiva de los participantes, esto es generado por una significativa falta de recursos y respaldos institucionales que dificultaban el trabajo intersectorial necesario para estos casos. Lo expresado, cuestiona el accionar fragmentado del Estado, evidenciando la necesidad de un abordaje intersectorial de la salud.

Por otra parte se hizo alusión sobre algunas cuestiones vinculados a la injerencia de la RAP de ASSE en temas asociados a las propuestas de capacitación y la gestión del centro de salud. Los señalamientos mencionados, fueron valorados negativamente ya que afectan la aplicabilidad de los desafíos emprendidos a nivel ministerial.

Por un lado, gran parte de los profesionales que trabajan en los centros de salud y policlínicas dependientes se han formado bajo otro paradigma de atención sanitaria, lo cual incide en la modalidad de trabajo adoptada y la relación que establecen con los usuarios. Por otro lado, las restringidas instancias de trabajo multidisciplinario tampoco suelen ser espacios donde se pueda remediar dichos desencuentros en pro de consensuar criterios asistenciales.

\footnotetext{
2. Programa dirigido a adolescentes entre los 10 y 19 años de edad, que residen en áreas de influencia de los efectores de salud del MSP y el Ministerio de Desarrollo Social. Su objetivo es la optimización de los derechos sexuales y reproductivos de los adolescentes, con énfasis en la promoción de la salud integral, atención del embarazo, y la parentalidad temprana (MIDES, 2007).
} 
Por lo tanto, frente a la ausencia de espacios de actualización profesional y capacitación oficialmente definidos, los consultados aludieron a la importancia del relacionamiento interpersonal a la interna del equipo de salud, compensando dicha carencia con el apoyo mutuo que se ofrecen.

Finalmente, en relación al subcomponente de las estructuras psicosocioculturales, se puede inferir que una de las variables que influye en el encuentro asistencial se relaciona con la valoración de los roles y responsabilidades esperables en esta etapa del ciclo vital (Amorín et al, 2006). En tal sentido, se produce el encuentro entre el estereotipo social de cada operador de salud sobre el "ser y estar" de un adolescente y el perfil real que suele presentar la usuaria del centro de salud convocado para el estudio. Resolver esta posible disociación, conforma un desafío revelador para el profesional de la salud si pretende construir un vínculo que posibilite y habilite el intercambio entre ambas partes.

En relación a esto, se pueden identificar una serie de documentos nacionales sobre las características sociodemográficas de la población adolescente y juvenil, donde se describe una tendencia diferencial en sus procesos de emancipación de acuerdo al nivel educativo alcanzado. En tal sentido, se observa indicadores claros respecto a una emancipación más temprana en quienes presentan menor nivel educativo. En estos casos, la transición a la adultez está marcada por la formación de su propia familia con hijos, siendo el embarazo el medio por el cual el adolescente suele adquirir estatus y reafirmar su ingreso hacía el mundo adulto (Ciganda, 2008; Filgueira, Kaztman y Rodríguez, 2005; MIDES, 2009; Varela, Pollero y Fostik, 2008). Algunas de las significaciones atribuidas a tal experiencia fueron comentadas al inicio del análisis, definiendo el mismo como la concreción del proyecto de vida de la mayoría de las adolescentes que concurrían al centro de salud.

Según lo sugerido por los consultados, se planteó la importancia de no relacionarse desde los estereotipos o prejuicios construidos culturalmente. Esto implicaría reconocer la naturaleza activa y protagónica del usuario en su proceso de atención, adoptando por parte del profesional de la salud una actitud de escucha y guía al servicio de la integración de saberes (Saforcada, 2008).

\section{Conclusiones}

La presentación y reflexión realizada sobre la información proporcionada por los participantes del estudio, permitió comprender los diferentes subcomponentes que integran el componente intersubjetivo general que opera en el encuentro asistencial dentro del marco ofrecido por el Espacio Adolescente del centro de salud involucrado.

El relevamiento de opiniones de los operadores de salud respecto a la temática elegida, permitió caracterizar la compleja constelación de subcomponentes que lo conforman, los cuales inciden en su posición frente a las usuarias, y por ende, en el trato que mantienen. En el contexto del embarazo y la maternidad en la adolescencia, esto adquiere un sentido y relevancia particular, ya que con frecuencia el centro de salud barrial, especialmente durante el primer año de vida del hijo/a de la adolescente, se convierte en el único punto de contacto con el afuera de su hogar (Garibotto et al., 2008; Pérez et al., 2010). Asimismo, el establecimiento de un vínculo satisfactorio por parte de la adolescente con el centro de salud, constituye una oportunidad para que integre otros espacios de atención, que le permita acceder a un abordaje integral de su salud y la de su hijo (MSP, 2009).

Se considera relevante introducir al cierre del artículo, una síntesis de las sugerencias brindadas por los participantes, las cuales podrían facilitar el encuentro entre los operadores de salud y las adolescentes embarazadas 0 madres que asisten a los servicios de salud. En primer lugar, se aludió a la relevancia de adoptar un enfoque basado en las fortalezas del sujeto, independiente de la edad del usuario, al momento de entablar el vínculo asistencial. En segundo lugar, el afianzamiento de las instancias multidisciplinarias parece ser una necesidad imprescindible para superar las barreras que suelen existir en el quehacer asistencial. En tercer lugar, asumir un enfoque constructivo sobre el embarazo en la adolescencia, desde donde sea visualizado como una posibilidad de entrelazar otros proyectos complementarios al mismo. En cuarto lugar, la necesaria construcción de una plataforma institucional que sea garante de un abordaje integral e intesectorial de la salud. Finalmente, se planteó la importancia de no relacionarse desde los estereotipos o prejuicios construidos culturalmente. 
Esto implicaría reconocer la naturaleza activa y protagónica del usuario en su proceso de atención.

La información obtenida a partir del análisis de los datos, tiene el potencial de favorecer la generación de insumos que guíen el protocolo de acompañamiento del embarazo y maternidad adolescente sugerido por salud pública. Este proceso de acercamiento y contemplación, tal vez permita, mayores puntos de encuentro entre el discurso y la praxis, y con ello, contribuir a alcanzar los compromisos asumidos por el nuevo modelo de atención integral de salud para adolescentes.

\section{Referencias}

Amorín, D., Carril, E., y Varela, C. (2006). Significados de maternidad y paternidad en adolescentes de estratos bajo y medio de Montevideo. En A. López Gómez (Coord.) Proyecto Género y Generaciones: reproducción biológica y social de la población uruguaya, ( $p$. 125-246). Uruguay: Trilce.

Ávila, L., y Tancredi, X. (2006) Diagnóstico de Situación de Salud de la zona de influencia de Jardines del Hipódromo [Monografía].Recuperado de http://www. medfamco.fmed.edu.uy/Archivos/monografias/Diagnostico\%20de\%20situacion\%20de\%20salud.pdf

Ciganda, D. (2008). Jóvenes en transición hacia la vida adulta: el orden de los factores ¿no altera el resultado? En C. Varela (coord.) Demografía de una sociedad en transición. La población uruguaya a inicios del siglo XXI., (p. 69 - 82) Montevideo: Trilce.

Comisión Económica para América Latina y el Caribe (1995). Panorama Social en América Latina. Recuperado de http://www.eclac.cl/

Comisión Económica para América Latina y el Caribe (2002). Panorama Social en América Latina. Recuperado de http://www.eclac.cl/

Comisión Económica para América Latina y el Caribe (2006). Panorama Social en América Latina. Recuperado de http://www.eclac.org/publicaciones/xml/0/27480/ pse_2006.pdf

Comisión Económica para América Latina y el Caribe (2008). Juventud y Cohesión Social en Iberoamérica. Un modelo para armar. Santiago de Chile: Naciones Unidas. Recuperado de http://www.eclac.org/ publicaciones/xml/2/34372/Juventud_Cohesion_Social_CEPAL_OIJ.pdf

Duncan, S. (2007) What's the problem with teenage parents? And what's the problem with policy? Critical social policy, 27 (3), 307-328.

Filgueira, F., Kaztman, R., y Rodríguez, F. (2005) Las claves generacionales de la integración y exclusión social: Adolescencia y juventud en Uruguay y Chile en los albores del siglo XXI. Revista Prisma, 21, 43-65.

Garibotto, G., González, G., Lorenzo, M., Methol, F., Monteiro, L., y Scarlatta, L. (2008) Tan embarazadas como valientes. Evaluación Cualitativa de los Acompañamientos Socioeducativos a adolescentes madres. Montevideo: Ministerio de Desarrollo Social, Programa Desarrolla PNUD, Cooperazione Italiana.
Gerstenblüth, M., Ferre, Z., Rossi, M., y Triunfo, P. (2009). Impacto de la maternidad adolescente en los logros educativos. Documentos de Trabajo,5, 1-18. Recuperado de http://decon.edu.uy/ publica/2009/0509.pdf

Hernández, R., Fernández, C., y Baptista, P. (2006) Metodología de la Investigación (4ª ed.). (p. 521-747) México: McGrawHill

Leiva, L. (2010) Maternidad y proceso de configuración identitaria en mujeres que fueron madres en la adolescencia: una aproximación desde el enfoque biográfico. (Tesis doctoral) Pontificia Universidad Católica de Chile.

Milles, M. \& Huberman, M. (1994) Qualitative Data Analysis ( $2^{\mathrm{a}}$ ed.). EE.UU: Sage Publications

Ministerio de Desarrollo Social, Infamilia (2007). La salud de los adolescentes y jóvenes uruguayos ¿protegida o a la intemperie? Recuperado de http://www.infamilia. gub.uy/page.aspx?1,7,194,O,S,0,

Ministerio de Desarrollo Social, Infamilia (2009) Encuesta Nacional de Adolescencia y Juventud 2008. Segundo Informe. Recuperado de http://www.inju.gub.uy/ innovaportal/file/9798/1/enaj_segundo_informe_cap1y2.pdf

Ministerio de Salud Pública (2007) Programa Nacional de Salud Adolescente. Recuperado de http://www.msp. gub.uy/andocasociado.aspx?5679,21552

Ministerio de Salud Pública (2009). Guías para el abordaje integral de la salud de adolescentes en el primer nivel de atención. Recuperado de http://www.msp.gub.uy/ uc_3886_1.html

Ministerio de Salud Pública (2010) Buenas Prácticas en salud sexual y reproductiva. Recuperado de http:// www.proyectodesarrolla.org/cat/publicaciones/

Ministerio de Salud Pública y Sistema Informático Perinatal (2011). Datos 2010 sobre Fecundidad Adolescente en Uruguay. Uruguay.

Pereira, J (2011a). Protección de Derechos y Acciones de Advocacy en el Trabajo con padres y madres Adolescentes. En G. Corbo y C. Guida, Mesas de Dialogo, Maternidad y Paternidad en la adolescencia. Problema: ¿para quién? Desafíos de las políticas públicas (p. 99 - 119). Recuperado de http://www. codajic.org/sites/www.codajic.org/files/MESAS\%20 DIALOGO\%20PATERNIDAD\%20MATERNIDAD\%20 ADOLESCENCIA.pdf

Pereira, J. (2011b). Academia, políticas e instituciones: problemas en las miradas adultas sobre la maternidad y paternidad en la adolescencia En A. Peregalli, Y. Sampietro, Maternidades, Paternidades y Adolescencias: relatos a viva voz. Buenos Aires: Novedades Educativas.

Peregalli, A., Ubal, M., y Méndez, J. (2011) Derecho a la Educación en madres y padres jóvenes y sus hijos: desafíos para la inclusión educativa. Recuperado de http://conenfor.edu.uy/innovaportal/file/19961/1/ libro_derecho_a_la_educacion.pdf

Pérez, P., Silva, M., y Escobar, M. (2010) Necesidades Educativas de madres adolescentes. Una mirada comunitaria. Montevideo: MIDES.

Reis Brandao, E. (2006). Gravidez na adolescência: un balanço bibliográfico. En M. L. Heilborn, E. Aquino, M. Bozon, y M. R. Knauth, O aprendizado da sexualidade. Reprodução e trajetória sociais de jovens brasileiros (p. 63 - 94), Brasil: Fiocruz. 
Saforcada, E. (1999). Psicología Sanitaria. Análisis crítico de los sistemas de atención de la salud. Buenos Aires: Paidós

Saforcada, E. (2008) Perspectiva ecológico-sistémica de la salud. En E. Saforcada y J. Castellá (Comp.) Enfoques conceptuales y Técnicos en Psicología Comunitaria, (p. 49 - 74). Buenos Aires: Paídos

Serbia, J. (2007) Diseño, muestreo y análisis en la investigación cualitativa. Hologramática, 7 (3), 123-146.

Setaro, M. y Koolhaas, M. (2008) Políticas de Salud. Montevideo: Comité de Coordinación Estratégica de Infancia y Adolescencia.
Stassen Berger, K. (2004). Psicología del Desarrollo. Infancia y Adolescencia ( $6^{\mathrm{a}}$ ed.). México: Panamericana.

Varela, C., Pollero, R., y Fostik, A. (2008) La fecundidad: evolución y diferenciales en el comportamiento reproductivo. En C. Varela (coord.) Demografía de una sociedad en transición. La población uruguaya a inicios del siglo XXI., (p. 35-67). Montevideo: Trilce.

Para citar este artículo:

Silva Laguardia, M. M. y Leiva Bahamondes, L. (2013). Abordaje sanitario del embarazo y la maternidad en la adolescencia. Perspectiva de los profesionales de la salud que trabajan en un centro de salud de la ciudad de Montevideo. Ciencias Psicológicas VII (1): 33 - 45. 